\title{
Juvenile polyposis syndrome
}

\author{
Wojciech Cichy ${ }^{1}$, Beata Klincewicz ${ }^{1}$, Andrzej Plawski
}

${ }^{1}$ Department of Pediatric Gastroenterology and Metabolic Diseases, Poznan University of Medical Sciences, Poznan, Poland

${ }^{2}$ Institute of Human Genetics, Polish Academy of Sciences, Poznan, Poland

Submitted: 1 March 2011

Accepted: 21 October 2011

Arch Med Sci 2014; 10, 3: 570-577

DOI: 10.5114 /aoms.2014.43750

Copyright (c) 2014 Termedia \& Banach

\section{Abstract}

Juvenile polyposis syndrome (JPS) is an autosomal dominant predisposition to the occurrence of hamartomatous polyps in the gastrointestinal tract. Diagnosis of JPS is based on the occurrence of numerous colon and rectum polyps or any number of polyps with family history and, in the case of juvenile polyps, their occurrence also outside the large intestine. The JPS is caused by mutations in SMAD4 and BMPR1A. Products of the SMAD4 gene are involved in signal transduction in the transforming growth factor $\beta$ pathway and BMPR1A protein is a receptor belonging to the family of transmembrane serine/threonine kinases. Both proteins are responsible for processes determining appropriate development of colonic mucosa. The JPS belongs to the group of hamartomatous polyposes. The hamartomatous polyposis syndromes constitute a group of diseases in which manifestations differ slightly and only molecular diagnostics gives the possibility of verifying the clinical diagnosis.

Key words: juvenile polyposis syndrome, BMPR1A gene, SMAD4 gene, juvenile polyposis.

\section{Introduction}

Proper development of a multicellular organism to its mature form as well as the subsequent maintenance of cell numbers at suitable quantitative and qualitative levels is of paramount importance for the functioning of this organism. In the course of evolution, higher organisms developed appropriate control mechanisms of cell division and differentiation as well as their migration, adhesion and death. The above-mentioned cell processes are, to a large extent, controlled by a relatively small number of signal transfer pathways, such as bone morphogenetic proteins (BMP), transforming growth factor $\beta$ (TGF- $\beta$ ), Wnt (wingless in Drosophila), Hedge $\mathrm{Hog}$, and Notch. The above signal pathways participate, among others, in the formation and correct maintenance of the systems of intestinal villi and crypts as well as in the development of highly specialised cells which are involved in the construction of the intestinal epithelium. The control of cell numbers as well as their proper differentiation is of considerable significance for the mucous membrane. In the gastrointestinal tract there occurs continuous loss of cells, which must be replenished, and frequent divisions and the influence of chemical compounds from cell DNA make this organ particularly prone to the occurrence of irregularities in the construction of the wall of the colon. The role of control mechanisms of cell proliferation and differentiation is very important as

Corresponding author:

Prof. Andrzej Plawski

Institute of Human Genetics

Polish Academy of Sciences

21 Strzeszynska St

60-479 Poznan, Poland

Phone: +48 616579215

E-mail: andp@man.poznan.pl 
any disturbance in the optimal operation of the components may have very negative effects on the appropriate functioning of the colon and, hence, the entire organism.

Among symptoms of abnormalities in the structure of intestinal mucosa is the occurrence of hamartomatous polyps. Hamartomatous polyps can be described as changes resulting from "incorrectly set" proper cells making up the lumen of the gastrointestinal tract leading to the development of structures of incorrect architecture. Juvenile polyps are hamartomatous polyps; single ones are observed in 1-2\% children as well as in teenagers [1]. Juvenile polyps are usually pedunculated, less frequently squat or flat. They have a smooth, glossy surface and may reach up to $4 \mathrm{~cm}$ in diameter. They are characterised by abundant lamina propria without smooth muscles and presence of widened, cyst-like glands [2]. Sometimes, stroma metaplasia towards cartilage or bones may occur. In the case of sporadic juvenile polyps, dysplastic changes are very rare and the latest reports rule out such changes, although in the case of polyps occurring in hereditary polyposis syndromes, the risk of dysplasia is considerable $[3,4]$.

\section{Disease characterisation}

Juvenile polyposis syndrome - JPS MIM \#174900 - is a genetically conditioned predisposition to the occurrence of juvenile polyps. The JPS was first described by McColl in 1964, although it was not until 10 years later that it was characterised comprehensively [5]. Like other hamartomatous polyposes, JPS is inherited in an autosomally dominated manner and from $20 \%$ to $50 \%$ of JPS cases follow a familial pattern. On average, the disease is diagnosed at 16 to 18 years of age and JPS polyps are usually found in the colon and rectum ( $80 \%$ of all cases), although sometimes they also occur in upper parts of the intestinal tract, such as the stomach and small intestine. The disease is characterised by heterogeneity with regard to age and symptom intensity, which is also observed among members of one family. It can be assumed that, apart from inheriting a mutation in one of the predisposition genes, a modifying impact of other genetic factors is also present, although they have not been identified so far.

The JPS diagnosis is based on the identification of at least one of the following three criteria:

- numerous juvenile polyps ( 3 to 10 polyps) in the colon and rectum [6],

- any number of juvenile polyps in patients with diagnosed familial history of the disease, and

- juvenile polyps outside the colon (in the stomach or small intestine).

Sachatello, in 1964, distinguished the following three forms of juvenile polyposis:
- juvenile polyposis in infants - an unusually rare form of juvenile polyposis with very serious course of the disease and poor prognosis,

- general form of juvenile polyposis in which polyps can be present in any part of the gastrointestinal tract with burdened or unburdened family anamnesis,

- colon juvenile polyposis - the most common form of JPS limited to polyps present in the colon with burdened or unburdened family anamnesis.

Patients with JPS are frequently observed to suffer from other congenial diseases such as intestinal malrotations and Meckel diverticula in the gastrointestinal tract. Congenial diseases of the heart, central nervous and of the urinary-sexual systems have also been reported. Other frequent symptoms include the occurrence of finger clubbing, macrocephaly, hypertelorism as well as cleft lips and palate [7-9].

Patients with JPS were also reported to suffer from the coexistence of symptoms typical for juvenile polyposis together with symptoms of equally rare Osler-Weber-Rendu syndrome (hereditary hemorrhagic telangiectasia-HHT). At the present time, the occurrence of features of these two diseases is defined as a separate syndrome of juvenile polyposis with hereditary haemorrhagic telangiectasia (MIM \#175050, juvenile polyposis/ hereditary haemorrhagic telangiectasia syndrome - JPHT). Skin telangiectasia, epistaxis, intracranial haemorrhage, development of pulmonary arteriovenous fistulas, brain cavernous angioma and haemangioma are among the most frequent symptoms of hereditary hemorrhagic telangiectasia in patients with JPHT syndrome. In addition, the occurrence of symptoms characteristic for hereditary hemorrhagic telangiectasia is diagnosed earlier in these patients than in the case of the isolated HHT form [10].

The risk of neoplastic transformations of juvenile polyps varies and it is assumed that sporadically occurring juvenile polyps do not pose such risk but in JPS patients the situation is quite different, since the risk of tumour development is considerable [11-13]. According to literature data, the risk of cancer development of the gastrointestinal tract is assessed at the level of $9 \%$ to $50 \%$ [12, 14]. It is estimated that colon tumour, which is the most common tumour of the gastrointestinal tract, develops in one fifth of patients suffering from juvenile polyposis syndrome. Occurrence of gastric, duodenal and pancreatic tumours has also been described $[12,15]$.

On the basis of long-term investigations of juvenile polyposis in a number of multi-generation families a phenomenon of anticipation was observed, i.e. the occurrence of pathological symptoms earlier and earlier and with increasing in- 
tensity in successive generations. Howe reported a six-generation family in which, from generation to generation, he observed the lowering of the occurrence of the first symptoms of the disease and, in some members of the family, co-occurrence of juvenile polyps together with changes typical for non-specific intestinal inflammations [15]. Nevertheless, it seems that, at least in part, the above-mentioned anticipation phenomenon may be associated with the increased awareness of patients in whose families a hereditary disease occurs, resulting in an earlier diagnosis and treatment.

\section{Structure of BMPR1A and SMAD4 genes and function of their protein products}

The development of juvenile polyposis is associated with mutations in one of two genes connected with the TGF- $\beta$ /BMP signal pathway. These genes are SMAD4 and BMPR1A. In 1998, the first gene associated with the predisposition to juvenile polyposis was mapped in chromosome 18 in the q21 region as a result of linkage analysis from a large family from lowa and it turned out to be the gene SMAD4 [15]. This report was confirmed by a number of identified germline mutations, including in a gene in patients with JPS, although mutations were diagnosed only in a small proportion of patients [11]. The locus of the second gene conditioning JPS was identified in 2001. Following linkage analysis comprising families from North America, SMAD4 and BMPR1A genes were identified $[16,17]$.

The $M I M^{*} 601299 ;$ Bone Morphogenetic Protein Receptor, Type IA (BMPR1A) gene is situated in chromosome 10 , in the q22-23 region $[18,19]$. The pseudogene of the BMPR1A gene is located in chromosome 6 . The BMPR1A gene is made up of 11 exons and has 20 introns equipped in classical dinucleotide GT donor and AG acceptor sites/places. The BMPR1A gene transcript consists of 3613 nucleotides. Following transcription, 12 different mRNA may develop - 10 as a result of alternative assembly and 2 forms without deleted introns. The BMPR1A gene codes the 532 amino acid transmembrane protein which acts as a type I receptor for bone morphogenic protein (BMP) ligands [20]. The protein BMPR1A gene product of $60 \mathrm{kDa}$ size consists of three main domains. An extracellular domain with a signal sequence is situated at the amine end of the protein. In addition, the extracellular domain closes the ligand binding domain and it is also the site of glycosylation. The mature $57.6 \mathrm{kDa}$ protein develops following cutting off of the signal sequence. BMPR1A binds with a number of ligands, including BMP2, BMP4 and BMP7, and it exhibits, in relation to other BMP type I receptors, certain affinity differences to various BMP ligands. For instance, BMPR1A shows lower affinity to the BMP7 ligand than to ALK6. As a homodimer, BMPR1A interacts with other homodimeric receptors (BMPRII, ActRII, ActRIIB) and also forms heterodimeric complexes with the ALK6 receptor. It also interacts with such auxiliary receptors as ENGOGLIN by binding with the BMP2 ligand. On the other hand, the SMAD4 gene (MIM*600993; family member 4) is a suppressor gene which is located on chromosome 18 in the 21.1 region [21]. It is made up of 11 exons and its genomic sequence includes $50 \mathrm{bp}$. Transcription may lead to the development of up to 18 different transcripts which arise as a result of alternative assembly as well as two forms without excised introns. The mRNA comprises 3197 nucleotides and codes a protein consisting of 552 amino acids. The protein product of this gene is an exceptionally important constituent of the transcriptional complex of many genes [22]. SMAD4, in contrast to the remaining SMAD, is not activated by phosphorylation but acts as an intracellular mediator. SMAD4 protein is made up of two globular domains. Similarly to the receptor SMAD, SMAD4 possesses two highly conservative domains: $\mathrm{MH} 1$ and $\mathrm{MH} 2$ (Mad homology domain). Both domains are connected through a coupling region. At the SMAD4 amine end, there is an $\mathrm{MH} 1$ domain characterised by a hairpin structure which shows DNA binding activity. On the other hand, the $\mathrm{MH} 2$ domain found at the carboxyl end is also present in the remaining SMAD. The MH2 domain is responsible for the interaction with proteins which take part in the complex translocation into the nucleus as well as with DNA binding co-factors [23]. The SMAD4 coupling region has a leucine-rich nuclear export signal (NES) recognised by CMR1. SMAD4 interactions with phosphorylated R-SMAD mask NES, thus protecting SMAD4 against its recognition by the nuclear export factor, CMR1 and export from the nucleus. SMAD4 export becomes possible only after R-SMAD dephosphorylation and complex dissociation.

Signal transfer along the TGF- $\beta$ /BMP pathway plays an important role during intestinal development as well as later maintenance of homeostasis of tissues which form it. The TGF- $\beta$ /BMP cytokinins are synthesised and secreted by various cell types in which they play significant roles in a wide range of cellular processes. Signal transfer requires two types of transmembrane receptors having serine-threonine kinase properties: type I and type II receptors. The BMP ligand binding to receptors causes the development of a heterotetrameric complex in which the activated type II receptor causes phosphorylation of the so-called $\mathrm{GS}$ region rich in serine and threonine located at 
the N-end part of the type I receptor. The signal is then transferred further from the cytoplasm to the nucleus by means of SMAD proteins (Figure 1). In the situation when the ligand happens to be missing, small FKB12 and FKB 12.6 proteins bind to the GS region, enforcing catalytic inactivation of the conformation of the receptor I kinase domain. The activity of the BMPR1A receptor is regulated by a number of proteins. Its activity was observed to increase, among others, in the presence of glycosylphosphatidylinositol. On the other hand, $\mathrm{BAMBI}$ - an inhibitor of pseudoreceptors BMP and actin - leads to decreased activity of the BMPR1A receptor and prevents signal transfer with the participation of this receptor.

The activation of the receptor leads to phosphorylation of SMAD and R-SMAD receptor proteins. Receptor SMADs at the carboxyl end of the polypeptide chain have a Ser-X-Ser motif which undergoes phosphorylation through the activated type I receptor. Further signal transfer, apart from the receptor SMADs, is accomplished with the assistance of two classes of SMAD proteins: inhibitor SMADs (I-SMADs) as well as SMADs binding with the receptor SMADs irrespective of the ligand type that activated the receptor (Co-SMADs). The SMAD proteins interact with one another in order to transfer signals. In the case of signal transfer by BMP receptors, receptor SMAD, SMAD 1, SMAD 5 and SMAD 8 are activated. Phosphorylated R-SMADs bind with Co-SMADs, i.e. SMAD4. The complex created in this way passes into the nu- cleus where it takes part in the expression of many genes as either a positive or a negative change regulator. Both activation and repression require the participation of the same SMAD proteins, whereas cell-specific interaction with factors which act as co-activators or co-repressors leads to an appropriate response. The SMAD4 complex with the receptor SMADs binds with DNA through the $\mathrm{NH} 1$ domain recognising the GTCTAGAC DNA palindrome sequence [24]. Such a SMAD binding sequence, known as Smad binding element (SBE), is frequently observed among genes which undergo expression in the presence of TGF- $\beta$ /BMP ligands. The SBE sequence GTCTAGAC can be found in the genome, on average, every $1024 \mathrm{bp}$. This gives, at least, one such place in the regulatory region of every medium-sized gene. In the literature, three mechanisms of transcription regulation in the promoter or enhancer by the SMAD or other transcription factors have been described. The first method involves binding of the active complex of receptor SMAD from SMAD4 with the transcription factor and this multi-molecular complex binds with the recognised DNA sequence. The second mechanism consists in separate binding of SMAD and co-factor with DNA but it is only the interaction of these proteins that stabilises enhancer properties. The last method of regulation involves independent binding of SMAD and the additional factor to a specific place in DNA. They act separately but in a synergistic manner $[25,26]$.

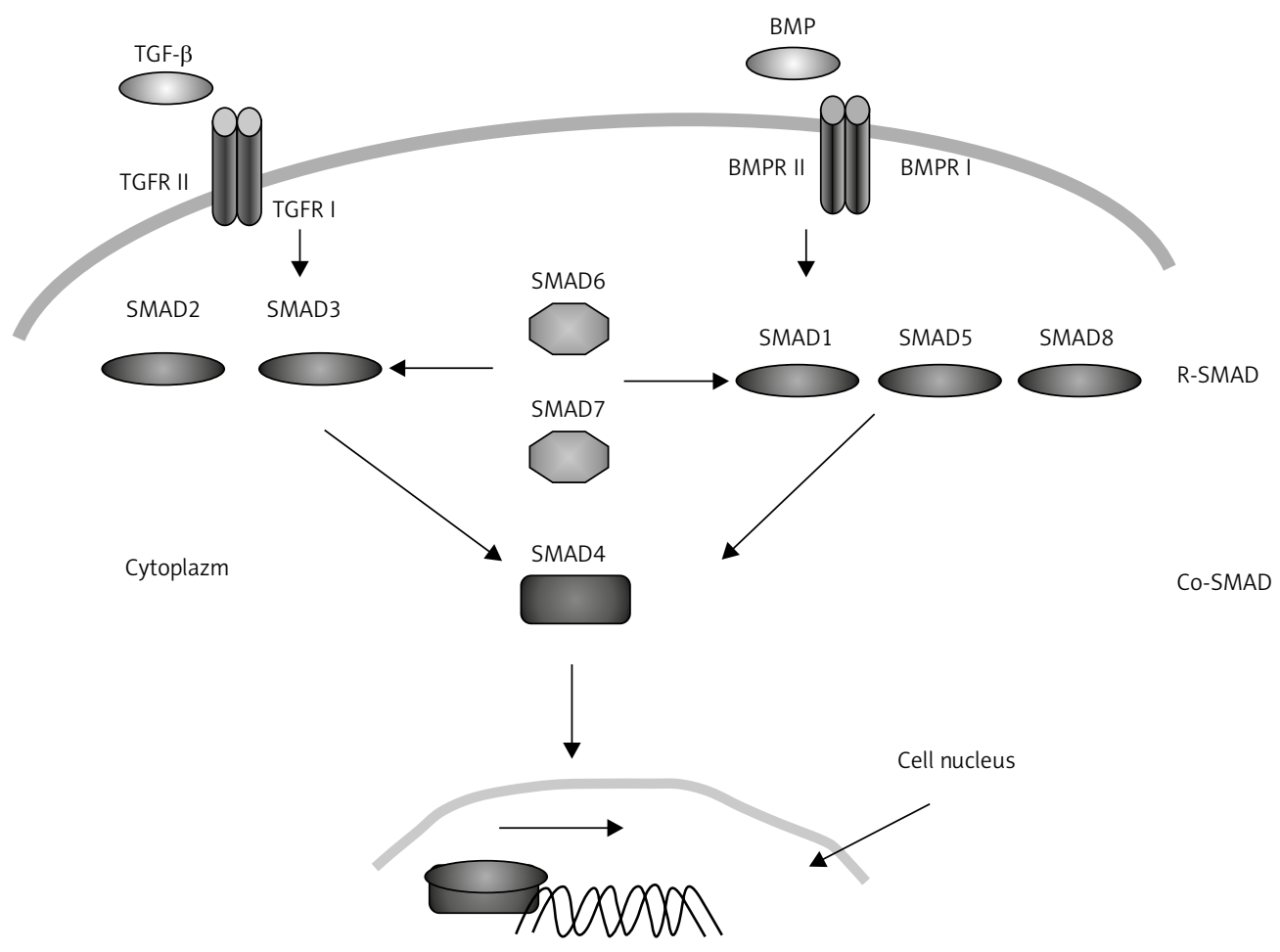

Figure 1. TGF- $\beta$ /BMP pathway 


\section{Mutation of BMPR1A and SMAD4 genes}

Mutations leading to the occurrence of juvenile polyposis are identified in two genes: BMPR1A and SMAD4 $[11,15,16]$. Mutations in the BMPR $1 A$ gene are diagnosed in approximately $20 \%$ of patients with juvenile polyposis and with similar frequency such mutations are identified in the SMAD4 gene.

Forty-three types of BMPR1A gene mutations were deposited in the Human Mutation Database at the Institute of Medical Genetics in Cardiff. Most often, these were nucleotide changes generating a stop codon (nonsense) or leading to amino acid changes (missense). These mutations are distributed evenly in the entire gene sequence. Five other mutations were reported at the gene assembly: two in intron 1, and one in each of introns 3, 4 and 5. Small deletions, most often identified between codons 224 and 359, constitute a considerable proportion of mutations in the BMPR1A gene. There are also reports in the literature of BMPR1A gene deletions together with a simultaneous deletion of the PTEN gene responsible for the development of hamartomatous polyps in Cowden syndrome.

Up to now, 40 mutations leading to juvenile polyposis as well as 5 mutations causing JPS with hereditary hemorrhagic telangiectasia have been described in the SMAD4 gene. From among all mutations in the SMAD4 gene, 17 included point mutations changing the nucleotide and, consequently, resulting in the development of a stop codon or causing a change in the coded amino acid into another one. Three point mutations in codons 361, 533 and 534 located within the $\mathrm{MH} 2$ domain were diagnosed in patients with JPHT. Among frequent mutations described in patients with JPS are small deletions; so far 14 such cases have been diagnosed. In addition, the mutation database also includes 6 insertions as well as one mutation in the donor place of gene assembly in intron 10 and one large 23-nucleotide deletion (SMAD4 c. 1343-1365).

Mutations described so far have been of heterogenic nature with the exception of mutation c.1244-1247delAGAC in exon 9 of the SMAD4 gene. This mutation is located in a hot spot, in a region containing four dinucleotide $A G$ repeats and one reverse repeat. The mechanism of its development is attributed to a polymerase slippage or to the presence of a palindrome sequence leading to unlooping of a fragment which undergoes deletion [27].

A high proportion of large genomic mutations both in BMPR1A and SMAD4 genes are diagnosed in patients with juvenile polyposis. The identification of large changes in these genes resulted in the increase of mutation detectability to $60 \%$ [28]. In addition, cases have been reported in the literature describing simultaneous deletions of two genes responsible for the occurrence of different syndromes of hamartomatous polyposis, namely the BMPR1A gene (juvenile polyposis) and the PTEN gene (Cowden syndrome) [29].

\section{Phenotype-genotype correlation vs. differentiation diagnostics}

There are scarce reports in the literature dealing with the phenotype-genotype correlation regarding juvenile polyposis. In part, this is due to small-numbered groups available to researchers as well as to the fact that at early stages of the disease of hamartomatous polyposis not all symptoms of the disease need to occur and, frequently, the symptoms that do occur may not be unambiguous.

In one of the first reports concerning genotype-phenotype correlations of both sporadic and familial JPS, it was reported that, among mutation carriers, a higher frequency of tumours of the gastrointestinal tract of familial course in one of the predisposition genes (SMAD4 or BMPR1A) was observed. In studies carried out by Sayed, a later age of diagnosis, more cases of the disease characterised by the familial course, and a greater number of polyps were observed in patients with mutations in any of the predisposition genes. Mutations in SMAD4 were correlated with polyps in higher segments of the gastrointestinal tract. The majority of them were massive gastric polyps. Therefore, in the case of patients with a diagnosed SMAD4 gene mutation, more frequent endoscopic examinations of the upper part of the gastrointestinal tract are recommended. Similar correlations in carriers of BMPR1A gene mutations were not ruled out.

The c.1244-1247delAGAC mutation in the hot spot of the SMAD4 gene leads to a more serious course of JPS with numerous cases of polyps located in the stomach and intestines as well as tumours in these organs [27].

Literature data about the occurrence of $\mathrm{HHT}$ symptoms in mutation carriers in the SMAD4 gene appear to indicate that patients with JPS, in particular, carriers of mutations in the SMAD4 gene, should be monitored regarding the occurrence of hereditary haemorrhagic telangiectasia syndromes such as aortic aneurysm or pulmonary thrombosis [30]. Similarly, it is believed that HHT patients should be diagnosed regarding the occurrence of juvenile polyps in the gastrointestinal tract $[10,28]$.

Unequivocal diagnosis of juvenile polyposis may sometimes be difficult in certain cases since juvenile polyps also occur in such hamartomatous syndromes as Cowden syndrome (CS), BannayanZonana syndrome (BZS) as well as in mixed polyposis (HMPS). The above-mentioned syndromes, 
especially in early phases of disease development, need not necessarily exhibit traits clearly distinguishing individual diseases, making diagnosis difficult. Both Cowden and Bannayan-Zonana syndromes arise as a result of mutations in the PTEN suppressor gene (with dual protein and lipid phosphatase activity). On the other hand, in patients with mixed polyposis of the second type, mutations in the BMPR1A gene were diagnosed. In the case of CS, symptoms include multiple polyps of hamartomatous type, macrocephaly as well as a higher risk of development of both benign and malignant tumours of the thyroid gland, breast, endometrium and skin [31]. In the case of the Bannayan-Zonana syndrome, apart from juvenile polyps, we can also observe macrocephaly, lipomata, mottled penis and vascular moles [32]. The HMPS patients are characterised by the presence of juvenile polyps which, together with polyps of adenoma and hyperplastic types, are found in the gastrointestinal tract. Cases were described in the literature of mutations in the PTEN gene in patients with juvenile polyps but the diagnosis claiming that those were cases of juvenile polyposis was questioned by other researchers because of the presence in adult patients of heterogenic thyroid nodules of laryngological tumours characteristic for Cowden syndrome [33]. It is generally maintained that, in the case of children, only several percent of CS clinical features are detectable before the $15^{\text {th }}$ year of life [2]. In addition, it should be remembered that at simultaneous deletion of $B M P R 1 A$ and PTEN genes, clinical features of JPS can coexist together with symptoms characteristic for Cowden syndrome. Initially, simultaneous deletion of BMPR1A and PTEN genes was attributed to patients with a severe course of infant juvenile polyposis, but at the present time it is assumed that the deletion of the $10 q 23$ region which contains these two genes is associated with a severe or moderate phenotype of the disease [29].

\section{Molecular diagnosis vs. medical procedures}

In genetic counselling preceding genetic tests, special emphasis should be paid, firstly, to the question whether pedigree analyses were carried out and, secondly, to obtaining information regarding clinical symptoms of the syndrome in the examined patient. Prior to carrying out examinations, each patient or his/her legal custodian should be informed about benefits and limitations resulting from the applied genetic tests. In addition, the patient should also be made aware of consequences of further medical care associated with positive or negative results of molecular examination. The genetic testing may be performed after expression by the patient of informed consent to the test. In the case of adolescents the decision about predispositional testing has to be made by parents or legal guardians.

Molecular diagnostics of juvenile polyposis involves seeking mutations primarily in two predisposing genes, namely SMAD4 and BMPR1A. It is only when no mutations in these genes are determined that molecular analysis of other genes associated with the occurrence of juvenile polyps should be performed. The use of molecular diagnostics in persons from the group of increased risk is of key importance because it makes presymptomatic recognition of mutation carriers possible.

The initial material that allows researchers to perform molecular studies is DNA isolated from nuclear cells of the peripheral blood. In SMAD4 and $B M P R 1 A$ genes, changes are of heterogenic nature; therefore, initially, screening analyses are carried out and, once the site of mutation has been located, direct nucleotide sequence analysis is conducted in order to assess the character of these changes. Screening methods comprise, mainly, techniques based on the detection of changes identified thanks to differences in migration of DNA fragments in the polyacrylamide gel caused by the mutation in the examined fragment. At the Institute of Human Genetics (IHG) of the Polish Academy of Sciences (PAS) in Poznan in which molecular diagnosis of hamartomatous polyposis is carried out, two techniques are employed. Their basis is amplification of the DNA fragment followed by denaturation of PCR products with the aim to obtain single-stranded fragments in single strand conformation polymorphism (SSCP) analysis or heteroduplex strands, mutated and correct in HD analysis [34, 35]. Among patients with juvenile polyposis, large genomic mutations constitute a significant proportion. On the other hand, the use of the multiplex ligation-dependent probe amplification (MLPA) technique allows complex analysis of exon deletions and duplications or of entire genes. This technique consists in the evaluation of the relative number of copies of duplicated DNA fragments and their comparison with control sequences located in other chromosomes. Probes employed in MLPA are designed in such a way as to allow simultaneous analysis of several dozens of fragments in one analysis. In IHG, analyses of large deletions in patients with JPS are carried out using the MLPA SALSA KIT P158-A1 Juvenile Polyposis (MRC-Holland), which allows detection of deletions or duplications in SMAD4, BMPR1A and PTEN genes [36]. The MLPA SALSA KIT P158-A1 includes probes for each exon of SMAD4, BMPR1A and PTEN genes. In the case of BMPR1A, the kit contains probes for all but one exon from this gene. The missing probe is that for exon 7 . This situation is due to the presence in chromosome 6 of the human genome of a pseudogene of identical 
sequence as that of exon 7 of the BMPR1A gene. That is why several probes in introns were designed. All in all, the kit for analysing large changes in patients with juvenile polyposis contains 46 probes. After probe ligation and the amplification reaction, the obtained products which are 110 to 472 nucleotides long are separated on a capillary sequencer.

In the situation when the mutation in a given proband is known, the identified mutation is further treated as a marker mutation within a given family. In such cases, a direct analysis can be applied [37].

In the process of mutation searching, some problems must be taken into consideration, such as the efficiency of mutation screening methods. The SSCP and HA methods allow detection of over $90 \%$ of mutations but not all. Sequencing of the whole coding sequence of predisposition genes is very expensive. A lower level of mutation detection is justified by cost effectiveness. In the case of MLPA, which is not a diagnostic method, the results may be disturbed by sequence variations in the probes of target sequences and by the condition of DNA sample as well. The exons with a lower level of signal have to be sequenced to exclude variation in the probe target DNA sequence. The best solution is to recognize the whole deleted sequence but it may be hampered if the deletion encompasses thousands of base pairs.

The expression 'mutation carrier' assumes the possibility of identification of a group of high risk and elimination from further investigations persons who are not mutation carriers. Secondary preventive treatment conducted in the group of mutation carriers makes early recognition of the disease and avoidance of malignant transformation possible. In addition, thanks to early performance of polypectomies, it is possible to avoid surgical intervention or at least to restrict its range.

At the present time, it is recommended that persons with the risk of occurrence of juvenile polyposis syndrome should undergo endoscopic examinations every year from the $15^{\text {th }}$ year of life or earlier if disease symptoms occur [38]. When only some polyps are identified, polypectomy is performed [39]. On the other hand, when polyps of high degree of dysplasia or polyps which cannot be removed in the course of endoscopic examination are detected and in the case of confirmed invasive malignant adenoma, then partial or total colectomy is carried out at about 20 years of age followed by annual endoscopic examinations of the colon [12]. Mutation carriers in predisposition genes should be monitored up to the age of 70 . In the case of examinations of the upper gastrointestinal segment of the tract, enteroscopic examinations using an endoscope capsule with simultaneous colonoscopy are recommended beginning at 25 years of age $[38,40]$. The above-mentioned examinations are recommended with higher frequency in carriers of the mutation in the SMAD4 gene than in carriers of the mutation in the $B M P R 1 A$ gene or persons from the risk group without an identified mutation, which is due to increased risk of polyp development in the upper segments of the gastrointestinal tract [41]. When only a few polyps are detected in the stomach, polypectomy is carried out, whereas when numerous, widespread polyps are diagnosed, partial or complete gastrectomy is performed.

The JPS is a well-known polyposis syndrome. However, further studies on the correct diagnosis and course of the disease need to be carried out. Wider use of molecular biology techniques will be helpful in this process.

\section{References}

1. Attard TM, Young RJ. Diagnosis and management of gastrointestinal polyps: pediatric considerations. Gastroenterol Nurs 2006; 29: 16-22.

2. Woodford-Richens K, Bevan S, Churchman M, et al. Analysis of genetic and phenotypic heterogeneity in juvenile polyposis. Gut 2000; 46: 656-60.

3. Chow E, Macrae F. A review of juvenile polyposis syndrome. J Gastroenterol Hepatol 2005; 20: 1634-40.

4. Huang SC, Erdman SH. Pediatric juvenile polyposis syndromes: an update. Curr Gastroenterol Rep 2009; 11: 211-9.

5. McColl I, Busxey HJ, Veale AM, Morson BC. Juvenile polyposis coli. Proc R Soc Med 1964; 57: 896-7.

6. Vaiphei K, Thapa BR. Juvenile polyposis (coli): high incidence of dysplastic epithelium. J Pediatr Surg 1997; 32: 1287-90.

7. Coburn MC, Pricolo VE, DeLuca FG, Bland KI. Malignant potential in intestinal juvenile polyposis syndromes. Ann Surg Oncol 1995; 2: 386-91.

8. Desai DC, Murday V, Phillips RK, Neale KF, Milla P, Hodgson SV. A survey of phenotypic features in juvenile polyposis. J Med Genet 1998; 35: 476-81.

9. Guillem JG, Smith AJ, Calle JP, Ruo L. Gastrointestinal polyposis syndromes. Curr Probl Surg 1999; 36: 217-323.

10. Gallione CJ, Repetto GM, Legius E, et al. A combined syndrome of juvenile polyposis and hereditary haemorrhagic telangiectasia associated with mutations in MADH4 (SMAD4). Lancet 2004; 363: 852-9.

11. Houlston R, Bevan S, Williams A, et al. Mutations in DPC4 (SMAD4) cause juvenile polyposis syndrome, but only account for a minority of cases. Hum Mol Genet 1998; 7: 1907-12.

12. Jarvinen H, Franssila KO. Familial juvenile polyposis coli; increased risk of colorectal cancer. Gut 1984; 25 : 792-800.

13. Jass JR, Williams CB, Bussey HJ, Morson BC. Juvenile polyposis: a precancerous condition. Histopathology 1988; 13: 619-30.

14. Hood B, Bigler S, Bishop P, et al. Juvenile polyps and juvenile polyp syndromes in children: a clinical and endoscopic survey. Clin Pediatr 2011; 50: 910-5.

15. Howe JR, Roth S, Ringold JC, et al. Mutations in the SMAD4/DPC4 gene in juvenile polyposis. Science 1998; 280: 1086-8. 
16. Howe JR, Bair JL, Sayed MG, et al. Germline mutations of the gene encoding bone morphogenetic protein receptor $1 \mathrm{~A}$ in juvenile polyposis. Nat Genet 2001; 28: 184-7.

17. Zhou XP, Woodford-Richens K, Lehtonen R, et al. Germline mutations in BMPR1A/ALK3 cause a subset of cases of juvenile polyposis syndrome and of Cowden and Bannayan-Riley-Ruvalcaba syndromes. Am J Hum Genet 2001; 69: 704-11.

18. Astrom AK, Jin D, Imamura T, et al. Chromosomal localization of three human genes encoding bone morphogenetic protein receptors. Mamm Genome 1999; 10 299-302.

19. Ide H, Saito-Ohara F, Ohnami S, et al. Assignment of the BMPR1A and BMPR1B genes to human chromosome 10q22.3 and 4q23-->q24 byin situ hybridization and radiation hybrid map ping. Cytogenet Cell Genet 1998; 81: 285-6.

20. Elliott RL, Blobe GC. Role of transforming growth factor Beta in human cancer. J Clin Oncol 2005; 23: 2078-93.

21. Howe JR, Ringold JC, Summers RW, Mitros FA, Nishimura DY, Stone EM. A gene for familial juvenile polyposis maps to chromosome 18q21.1. Am J Hum Genet 1998; 62: 1129-36.

22. Zhang Y, Musci T, Derynck R. The tumor suppressor Smad4/DPC 4 as a central mediator of Smad function. Curr Biol 1997; 7: 270-6.

23. Whitman M. Smads and early developmental signaling by the TGFbeta superfamily. Genes Dev 1998; 12: 2445-62

24. Zawel L, Dai JL, Buckhaults P, et al. Human Smad3 and Smad4 are sequence-specific transcription activators. Mol Cell 1998; 1: 611-7.

25. Massague J, Seoane J, Wotton D. Smad transcription fac tors. Genes Dev 2005; 19: 2783-810.

26. Massague J, Wotton D. Transcriptional control by the TGF-beta/Smad signaling system. Embo J 2000; 19 1745-54.

27. Howe JR, Shellnut J, Wagner B, et al. Common deletion of SMAD4 in juvenile polyposis is a mutational hotspot. Am J Hum Genet 2002; 70: 1357-62.

28. Aretz S, Stienen D, Uhlhaas S, et al. High proportion of large genomic STK11 deletions in Peutz-Jeghers syndrome. Hum Mutat 2005; 26: 513-9.

29. Salviati L, Patricelli M, Guariso G, et al. Deletion of PTEN and BMPR1A on chromosome 10q23 is not always as sociated with juvenile polyposis of infancy. Am J Hum Genet 2006; 79: 593-6; author reply 6-7.

30. Iyer NK, Burke CA, Leach BH, Parambil JG. SMAD4 mutation and the combined syndrome of juvenile polyposis syndrome and hereditary haemorrhagic telangiectasia. Thorax 2010; 65: 745-6.

31. Pilarski R, Eng C. Will the real Cowden syndrome please stand up (again)? Expanding mutational and clinical spectra of the PTEN hamartoma tumour syndrome. J Med Genet 2004; 41: 323-6.

32. Gujrati M, Thomas C, Zelby A, Jensen E, Lee JM. Bannayan-Zonana syndrome: a rare autosomal dominan syndrome with multiple lipomas and hemangiomas: a case report and review of literature. Surg Neurol 1998; 50: $164-8$

33. Stanich PP, Owens VL, Sweetser S, et al. Colonic polyposis and neoplasia in cowden syndrome. Mayo Clinic Proceedings Mayo Clinic 2011; 86: 489-92.

34. Kaczmarek M, Hoppe-Gołębiewska J, Słomski R. Wykry wanie mutacji punktowych i polimorfizmu DNA metodą SSCP. In: Analiza DNA, teoria i praktyka [Polish]. Slomski R (ed.). Wyd. Uniwersytetu Przyrodniczego, Poznan 2008; 195-202
35. Pławski A, Podralska M, Słomski R. Wykrywanie mutacji metodą heterodupleksów. In: Analiza DNA, teoria i praktyka [Polish]. Słomski R (ed.). Wyd. Uniwersytetu Przyrodniczego, Poznan 2008; 203-8.

36. Pławski A, Kaczmarek M, Podralska M, Hoppe-Gołębiewska J, Słomski R. Wykrywanie delecji i duplikacji metodą MLPA. In: Analiza DNA, teoria i praktyka [Polish]. Słomski R (ed.). Wyd. Uniwersytetu Przyrodniczego, Poznan 2008; 225-9.

37. Słomski R, Pławski A. Sekwencjonowanie DNA - elektroforeza kapilarna. In: Analiza DNA, teoria i praktyka. Slomski R (ed.). Wyd. Uniwersytetu Przyrodniczego, Poznan 2008; 429-34.

38. Dunlop MG. Guidance on gastrointestinal surveillance for hereditary non-polyposis colorectal cancer, familial adenomatous polypolis, juvenile polyposis, and Peutz-Jeghers syndrome. Gut 2002; 51 Suppl 5: V21-7.

39. Teisseyre MRJ, Celińska-Cedro D, Woynarowski M, Dziki T, Hor I. Endoskopowe usuwanie polipów jelita grubego u dzieci - 28-letnie doświadczenie własne. Pediatr Współcz Gastroenterol Hepatol Żyw Dz 2007; 9: 251-3.

40. Yamao $\mathrm{T}$, Isomoto $\mathrm{H}$, Yamaguchi $\mathrm{N}$, et al. Magnified endoscopic observation using narrow-band imaging of periampullary adenoma in a patient with familial adenomatous polyposis. Med Sci Monitor 2009; 15: CS169-73.

41. Sayed MG, Ahmed AF, Ringold JR, et al. Germline SMAD4 or BMPR1A mutations and phenotype of juvenile polyposis. Ann Surg Oncol 2002; 9: 901-6. 